\title{
S28. Improvement of the quality of life in schizophrenia (supported by an educational grant from Lundbeck DK)
}

WHAT IS RELAPSE IN SCHIZOPHRENIA?

Professor Malcolm Lader.

Institute of Psychiatry, De Crespigny Park,

London, SE5 8AF, U.K.

Relapse can be defined as "the return of a disease after partial recovery". This definition is directly applicable to schizophrenic disorder. Relapse in this condition can be evaluated at the symptomatic, phenomenological and behavioural levels. Relapse impinges on inter-personal, social and occupational spheres of the patient's activity. In turn, relapse has wider implications for the family in general, for the provision of medical and social facilities and for health economics.

There are no generally accepted criteria for relapse but outcomes such as violence or suicide, extreme psychotic behaviour and rehospitalisation have been used. Less obvious criteria include major and minor symptom worsening and prodromata.

Relapse is a relative term and must take into account the following factors: The patient's condition before the original onset of illness: his level of functioning before the present episode; the severity of the relapse in terms of symptom severity, duration and interference with personal functioning; the appearance of any new symptoms or behaviour patterns; the type of treatment given prior to relapse, and hence relatively ineffective; the type of treatment given during relapse, and its efficacy.

Relapses often follow a pattern which may be fairly specific to each individual patient.

Social factors may have predictive power but these need validation in different patient samples.

\section{INTERMITTENT MEDICATION - AN ALTERNATIVE?}

W Gaebel

Department of Psychiatry, Heinrich Heine University Düsseldorf, Bergische Landstraße 2, D-40629 Düsseldorf, Germany

Neuroleptic maintenance medication is clearly effective for relapse prevention in schizophrenia. However, besides benefits for the majority of patients, there are aiso failures and/or serious risks for some patients (e.g. tardive dyskinesia). Since the risk-benefit ratio is often difficult to predict in the individual case, this has stimulated the search for modifications and alternatives to maintenance treatment. In particular, neuroleptic low-dose treatment strategies compare quite favorably with standard-dose treatment conceming relapse prevention and side effects.

Atternatively, on the basis of reports on prodromal symptoms preceding a relapse, early intervention, intermittent neuroleptic treatment strategies have been developed. However, all recently completed controlled two-year studies have not confirmed this strategy to be as effective as maintenance treatment in preventing relapse, atthough total drug exposure can be significantly reduced and social adjustment seems to be unaffected. As to the possible reasons for the failure in relapse prevention it has been shown that prodromal symptoms are no valid predictors of relapse (Gaebel et al. 1993). Although prodromal symptoms may be taken as potential warning signs of relapse early intervention treatment has not been proven successful. Moreover, the neurobiological consequences of intermittent treatment are unknown and may at least be harmful according to some clinical and animal studies demonstrating a higher incidence of movement disorders compared to continuous treatment.

Accordingly, intermittent treatment cannot be generally recommended. Instead, low-dose neuroleptic maintenance treatment combined with psychosocial interventions is still the treatment of choice for the majority of schizophrenic patients.

References: W Gaebel et al, Brit J Psychiatry 163 (suppl 21): 8-12 (1993) 
ORAL VS DEPOT ADMINISTRATION OF NEUROLEPTICS IN RELAPSE PREVENTION

J. Gerlach

St. Hans Hospital, Department P, Roskilde, Denmark

The introduction of depot neuroleptics 20 years ago was a major advantage in the maintenance treatment of chronic schizophrenia. Especially in patients with lack of judgement and insight, the depot formulation highly reduced the problems of non-compliance, relapse and rehospitalization. It is known that more than half of the schizophrenic patients are inclined to stop taking their antipsychotic medication. Besides the reliable drug administration, the wellrecognized advantages of depot neuroleptics include the avoidance of absorption problems and first pass metabolism, a relatively stable, low plasma drug level and a potentially lower level of side effects, including EPS.

Another advantage of depot neuroleptics is less recognized. By means of depot neuroleptics it is relatively safe to use the lowest effective dosage principle, i.e. to gradually decrease the dose over several months untill the appearance of mild prodromal symptoms and then raise the dose by one step. In case of zuclopenthixol decanoate it is possible to give a single injection of the zuclopenthixol acetate formulation (Acutard) in order to quickly overcome a symptom aggravation. With peroral neuroleptics the gradual dose reduction may lead the patient to stop taking the medication, without informing the doctor, and then suddenly go into a severe relapse. In case of depot, the patients remain under influence of the medication and develop a more mild and easily treated symptom exacerbation. Especially in these years with focus on the patients' subjective experience and attitude to the medication it is essential for each single patient to find the lowest dose possible, the individual dose, and not a standardized, sometimes relatively high dose based on plasma concentration monitoring.

SIDE EFFECTS OF ANTIPSYCHOTICS

WW Fleischhacker

Dept. of Biological Psychiatry, University of Innsbruck, Medical School, Anichstrasse 35 , A-6020 Innsbruck, Austria

The side effects of antipsychotics have always been a major concern for clinicians and the appreciation of their importance in the treatment of schizophrenia has increased steadily over the years. Epidemiological studies as well as trials of the prevention and treatment of antipsychotic side effects are the consequences of this

development. Results of these studies also have important implications for improving compliance in schizophrenic patients. Thus, side effects research has made the treatment of schizophrenia not only safer but also more effective. Next to a brief overview of some of the more important adverse events that have been recently studied, the side effect profiles of the new antipsychotics will be discussed in this context.
COST EFFECTIVENESS STUDIES IN THE EVALUATION OF MENTAL HEALTH SERVICES IN THE COMMUNITY: CURRENT KNOWLEDGE AND UNSOL VED PROBLEMS

D Goldberg

Institute of Psychiatry, London

This paper will review current knowledge on studies that have been carried out on the cost-effectiveness of the care of schizophrenic patients in community settings, and will conclude that care in the community is generally cheaper and often better than care in hospital settings. However, there are many expections to this generalisation, and the audience will be invited to address a number of unsolved problems:

* What are the best ways of addressing the needs of high dependency patients with severe mental illnesses?

* Which patients are best treated in hospital settings, and what is the size of this need?

* How do variations in the social ecology of a district affect the optimal configuration for mental illness services?

* How should community services respond to the needs of ethnic minorities? 